\title{
Melanoma: Surgery Subsequent to PDT-Is It Worth Doing? A 10-Year Pilot Trial Using Chlorin-Type Photosensitizer Bremachlorin ${ }^{\bullet}$
}

\author{
Evgeniy I. Volkov ${ }^{1}$, Andrei V. Reshetnickov²,3\#, Harrie Vink ${ }^{3}$ \\ ${ }^{1}$ State Budget Healthcare Facility "Sverdlovskiy Oblastnoy Oncologicheskiy Dispancer”, Ekaterinburg, Russia \\ ${ }^{2}$ AREV PHARM LLC., Moscow, Russia \\ ${ }^{3}$ RADA-PHARMA International B.V., Steenbergen, The Netherlands \\ Email: " a.reshetnickov@gmail.com
}

\begin{abstract}
How to cite this paper: Volkov, E.I., Reshetnickov, A.V. and Vink, H. (2017) Melanoma: Surgery Subsequent to PDT-Is It Worth Doing? A 10-Year Pilot Trial Using Chlorin-Type Photosensitizer Bremachlorin ${ }^{\circledR}$. Journal of Cancer Therapy, 8, 902-912. https://doi.org/10.4236/jct.2017.810079
\end{abstract}

Received: August 16, 2017

Accepted: October 29, 2017

Published: October 31, 2017

Copyright $\odot 2017$ by authors and Scientific Research Publishing Inc. This work is licensed under the Creative Commons Attribution International License (CC BY 4.0).

http://creativecommons.org/licenses/by/4.0/

c. (i) Open Access

\begin{abstract}
Bremachlorin $^{\circledR}$, also known as "Radachlorin ${ }^{\circledR}$ " and Photochlorin, a composition of 3 chlorophyll a derivatives in an aqueous solution [1], was initially introduced into the Russian and South Korean Pharmacopoeias with the corresponding marketing authorizations received in 2006 and 2008, on the basal cell skin carcinoma PDT protocol. Phase I-II clinical trials led in 2002-2005 showed no side effects, a good tolerability by patients and a high clinical anti-tumor efficacy. Further studies described in the present paper were fulfilled in accordance with a skin melanoma PDT + surgery combined technique protocol in 2006-2016. Resulting from the present trial, up to the $100 \%$ complete responses were achieved after resection and plastic surgery following PDT, with no melanoma regrowth or metastases during 5 and 10 -year observation periods. Conducting PDT one day prior to tumor dissection can guarantee to meet the ablastics principles at the subsequent surgery. At the same time, the PDT + surgery protocol can allow for dissecting the tumor with less distance from its edge, which seems to be important for the face localizations of tumors.
\end{abstract}

\section{Keywords}

Melanoma, Skin, PDT, Radahlorin, Radachlorin, Bremachlorin, Photochlorin, Laser, Cancer

\section{Introduction}

Melanoma is responsible for $80 \%$ mortality within all the skin oncopathology,

${ }^{\star}$ The research was done under grant \#2-2004 from the Natural Health Foundation (Westeinde 82, Noordwijkerhout 2211XR, The Netherlands, http://www.photoimmune.org/). 
with taking only $10 \%$ in the structure of these diseases.

From the PDT point, the $1^{\text {st }}$ generation photosensitizers (PSs) were not much efficient at treating thick or nodular tumors by PDT, because of a light penetration into them (1-3 $\mathrm{mm}$ at $630 \mathrm{~nm})$. Despite non-melanoma skin cancers (e.g. nodular BCC) were, nevertheless, with a certain success, treated by PDT as a single treatment using methylaminolevulinate [2], preceding works on melanoma were concentrated around resection followed by PDT, with illuminating the area inside or around the operation field. It served the purpose of removing either the remaining melanoma cells, or residual tumor and surrounding pre-melanoma cells and lesions [3].

Our phase II clinical studies dating back to 2003-2005 demonstrated a high anti-tumor activity of radahlorin-PDT with respect to, in particular, nodular forms of basal cell carcinoma of the skin: $84.5 \% \mathrm{CR}$ and $15.5 \% \mathrm{PR}+\mathrm{S}$ by the end of a 2-month effect evaluation period. One and a half year after the treatment, CR was present in $92.8 \%$ patients who had CR as evaluated after 2 months [4]. That unexpected result was achieved, probably, due to a deeper (up to $7 \mathrm{~mm}$ ) light penetration into tumors at $662 \mathrm{~nm}$ wavelength used to activate the second generation PS radahlorin. It encouraged us to investigate the reversed order of manipulations at treating melanomas: PDT followed by surgery, to improve the efficacy of the latter, as well as to reduce the occurrence of metastases after incomplete resections.

This paper represents a full report of pilot clinical trials for photosensitizer Bremachlorin in Russia (October 2006-October 2016), previously published in brief [5], performed in 1 hospital, aimed at exceeding the 10 year survival of patients with melanoma of the skin having combined treatment: PDT followed by surgery.

\section{Materials and Methods}

\subsection{Photosensitizer}

Chlorin $e_{6}$, chlorin $p_{6}$ and purpurin 5 precursors were produced by AREV PHARM LLC, Moscow, Russia.

Radahlorin $^{1}$ active pharmaceutical ingredient (API) as well as its finished formulation Bremachlorin ${ }^{\circledR}$ solution for intravenous infusions $0.35 \% 10 \mathrm{ml}$ (BC), also known in some countries as "Radachlorin ${ }^{\circledR}$ " and Photochlorin, was made by RADA-PHARMA International B.V. Steenbergen, The Netherlands, or by other EU producers on a contract basis.

$\mathrm{BC}$ is radahlorin $(5.00 \mathrm{~g})$ (containing total potassium salts of the 3 chlorins- $0.40 \mathrm{~g}$, and water-the rest), water for injections (up to $100.00 \mathrm{~g}$ ), and additives: $\mathrm{N}$-methyl-D-glucamine $(0.20 \mathrm{~g})$, potassium hydroxide and hydrochloric acid for the $\mathrm{pH}$-adjustment to $\mathrm{pH} 8.5 \div 10.5$.

\footnotetext{
${ }^{1}$ Here and further radahlorin means the pharmacopoeian name of the active pharmaceutical ingredient (API) being the non-proprietary name, while "Radachlorin ${ }^{\circledR}$ ", Bremachlorin (BC), Photochlorin relate to the finished formulations (pharmaceutical forms) made by different producers for various markets for humane application, and Oscirad-for veterinary usage.
} 
For radahlorin photoactivation $2.5 \mathrm{~W} 662 \pm 3 \mathrm{~nm}$ semiconductor lasers were employed.

\subsection{Photodynamic Diagnosis}

Photodynamic diagnosis (PDD) was performed using spectral diagnostic systems “LESA-01-Biospec” (BIOSPEC Laser Biospectroscopy Lab., Moscow, Russia).

In the majority of patients the tumor borders determined by PDD were considerably wider than the tumor borders determined visually.

The therapeutic ratio was $3-4$ at $0.5 \mathrm{mg} / \mathrm{kg}$ drug dose. Remarkably, the tumor fluorescence is stronger in $24 \mathrm{~h}$, than in $12 \mathrm{~h}$ p.i. without PDT. We think that it is connected with the photosensitizer (PS) photobleaching in the daytime with the consequent restitution of the PS properties at night time.

\subsection{Patients, Clinical Base and Sponsor}

Forty-eight patients having $\mathrm{T}_{1-3} \mathrm{~N}_{0} \mathrm{M}_{0}$ (Clark level of invasion) pigmented melanoma of the skin took part in the present study of BC with a 10-year follow-up. Half of them had surgery following PDT, and half of them-surgery only, according to the conventional protocol of resection. The pre-clinical studies were fulfilled beforehand [4] [6].

The photosensitizer was approved in Russia in October, 2006 for PDT of both erosive and nodular forms of basal cell cancer $\mathrm{T}_{1-4} \mathrm{~N}_{0} \mathrm{M}_{0}$ and was used in the present study off-label on live indications, with a patient written consent and in a combination with the radical removal of the tumor after PDT, with no proven regional or distant metastases. It was used as an i.v. solution or gel for topical application [4] [7].

The study was conducted at the specialized oncological center of a regional significance-State budget healthcare facility "Sverdlovskiy Oblastnoy Oncologicheskiy Dispancer", Ekaterinburg, Russia.

The medicine was provided by the patent holder (the EC states, South Africa, Australia, UK, New Zealand) and its producer, RADA-PHARMA International B.V., Steenbergen, The Netherlands.

The research was supported by a non-profit organization aiming at developing and promoting more efficient and less debilitating cancer therapies, the Natural Health Foundation (Noordwijkerhout, The Netherlands).

\subsection{Photodynamic Therapy}

A flowchart of the study design according to GCP (Good Clinical Practice) Protocol (Chart 1).

\subsection{Dose-Effect Study to Find the Most Efficient Drug Dose}

Purpose: to find the most effective regime for treating pigmented melanoma by PDT. 


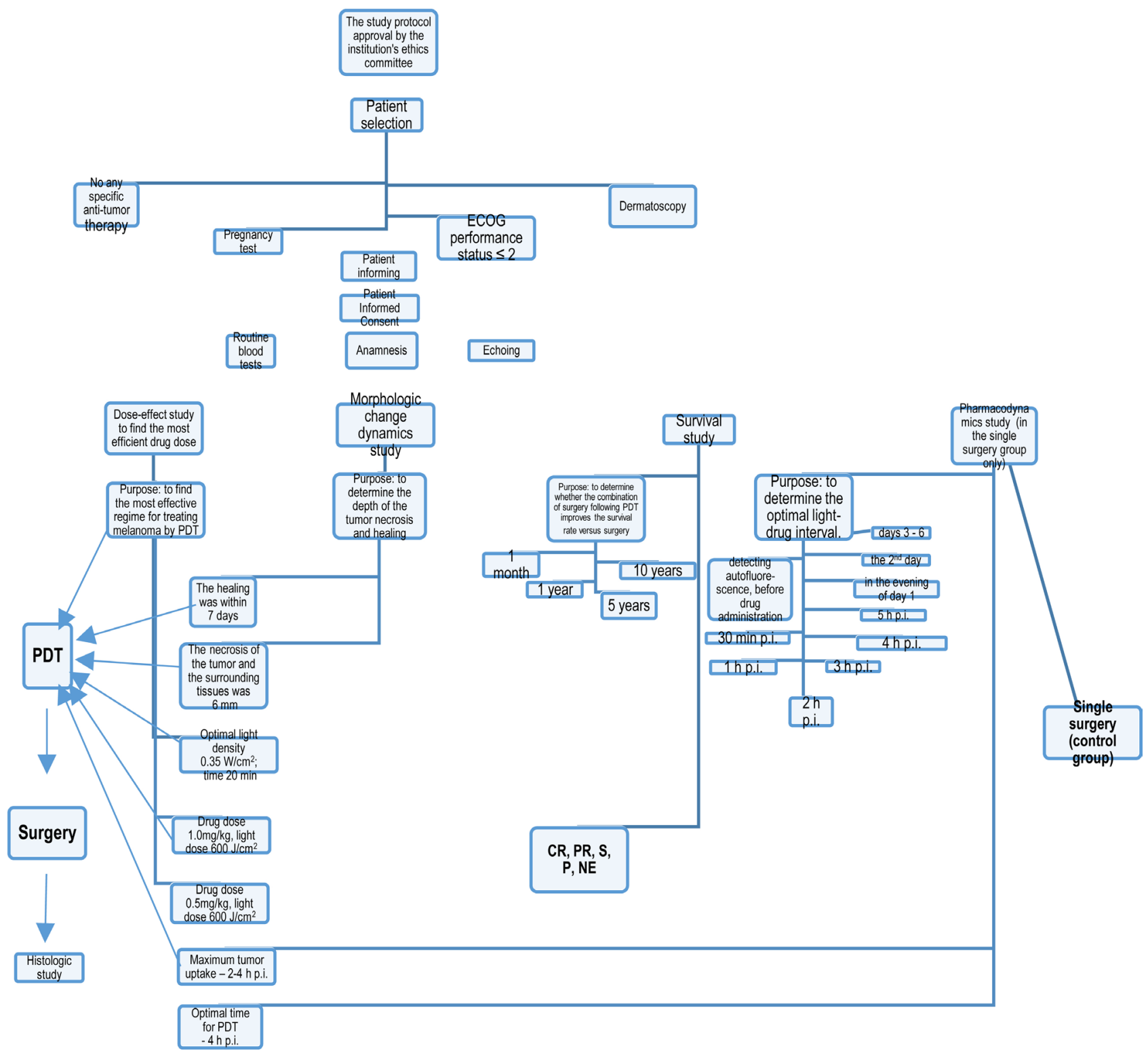

Chart 1. A flowchart of the study design according to GCP (Good Clinical Practice) Protocol.

1) Drug dose $0.5 \mathrm{mg} / \mathrm{kg}$, light dose $600 \mathrm{~J} / \mathrm{cm}^{2}$

2) Drug dose $1.0 \mathrm{mg} / \mathrm{kg}$, light dose $600 \mathrm{~J} / \mathrm{cm}^{2}$

The examination of the patients clinically and dermatoscopically was done on days $1,8,15,22,29$, months $3,6,9,12$, years $2-10$.

\subsection{Morphologic Change Dynamics Study}

Purpose: to determine the depth of the tumor necrosis.

Surgical removal of the tumor was done on day 1 or day 2 after PDT, by way of a dissection under endotracheal or intravenous narcosis.

Morphologic study showed:

a) the necrosis of the tumor and the surrounding tissues in $\mathrm{mm}$;

b) the curative pathomorphosis. 


\subsection{Survival Study}

Purpose: to determine whether the combination of surgery following PDT improves the survival rate versus single surgery on a 5 - and 10 -year basis.

\subsection{PDT + Surgery Combined Treatment}

Patient selection was carried out in complete accordance with the protocol, with meeting the following conditions:

- any specific anti-tumor therapy had to be absent before start of the study;

- result of pregnancy test carried out one week before start of the study had to be negative;

- patient's condition had to be satisfactory (ECOG performance status $\leq 2$ );

- the study had to be preceded by dermatoscopy for the verification of diagnosis;

- all the candidates for the study participation had to be informed about potential risks;

- informed consent was obtained from each patient;

- the study protocols conformed to the ethical guidelines of the 1975 Declaration of Helsinki as reflected in their priori approvals by the institution's human research committees.

Anamnesis was collected. The echoing of the neck, armpits, heart and abdomen, as well as tests for $\mathrm{HCV}, \mathrm{HBs}$, blood biochemistry, urine general test were routinely performed.

Primary single melanoma history of 0.5 - 6 years. The skin melanoma was diagnosed clinically and by dermatoscopy. The pigmented tumors located mainly on arms, face. Size of the tumors was $1.5-3 \mathrm{~cm}$ in diameter, irregular, occasionally under a scrub, sometimes represented by the lentigo maligna (surrounded by Hutchinson's melanotic freckle). Most of them were slightly exophytic. In 4 6 months directly preceding to visiting a physician the lesions became more pigmented, itching, growing rapidly exophytically, sometimes bleeding. There were no proven data for regional or distant metastases. BC was administered dropwise in $100 \mathrm{ml}$ saline for $30 \mathrm{~min}, 4 \mathrm{~h}$ prior to the laser irradiation. PDT was accomplished at $600 \mathrm{~J} / \mathrm{cm}^{2}$ (laser outputs-0.5 $-2.5 \mathrm{~W}$, surfacial power densities $-0.05-0.5 \mathrm{~W} / \mathrm{cm}^{2}$, optimally, 0.35 , as the most tolerable by the patients from the pain point), time of irradiation-5 - $60 \mathrm{~min}$, optimally, $20 \mathrm{~min}$.

Swelling and skin ischemia were seen right after PDT, with a light hyperemia around the illuminated zone and tumor color change to a lighter one. Tumor dissection was done in 2 - $3 \mathrm{~h}$ after PDT on day 1 in some patients, or on other days, according to the protocol. Plastic surgery was performed. A histologic study of the removed tumor was done to determine the level of invasion, type of melanoma and the depth of necrosis with a morphologic study. It also showed how radical the operation was done.

In the morphologic study, the ischemia of tissues and blood vessel thrombosis in the skin were always observed. The skin and underlying derma were mod- 
erately infiltrated, the skin having a blueish tint with multiple petechial spots found up to $6 \mathrm{~mm}$ in depth. The PDT tumor necrosis was detected to locate up to the depth of $3-6 \mathrm{~mm}$.

The same procedures save for PDT were performed for the $2^{\text {nd }}$ (control) group of the patients.

The patients stayed in the clinic for 1 week after the operation up to the per prima healing.

Efficacy of the treatment in both groups was evaluated based on the following WHO criteria (UICC) after 1, 5, 10 years:

- complete remission: absence of any signs of disease;

- partial remission: recurrence with metastases during the period one month 5 years after treatment;

- partial remission with stabilization: recurrence without metastases during the period one month - 5 years after treatment;

- disease progression: recurrence with metastases and death of patient within 1 year.

\section{Results}

\subsection{Safety}

Safety study showed no side effects and a good tolerability of PDT with BC followed by surgery by patients, save for moderate pain, depending on individual sensitivity, tumor localization and irradiation field. There was no normal skin/ subdermal tissue damage after both laser and daylight exposure.

\subsection{Pharmacodynamics Data}

Purpose: to determine the optimal light-drug interval. A fluorescence study (drug dose $0.25 \mathrm{mg} / \mathrm{kg}$ in the single surgery group only, involving 7 patients for this study):

Seven patients from 24 undergoing surgery only were injected a small dose of the PS for its accumulation measurements by detecting its fluorescence in:

1) the skin of the hand,

2) 4 spots in the vicinity of the tumor,

3) over the tumor,

4) on the tumor/normal tissue border.

Fluorescence detection was performed according to a specified method using standard BC samples (with a known concentration, and a calibration curve):

1) detecting autofluorescence to further distract it, immediately before administering the PS,

2) 30 min p.i.

3) 1 h p.i.,

4) 2 h p.i..

5) $3 \mathrm{~h} \mathrm{p.i.}$

6) 4 h p.i. 
7) 5 h p.i.

8) in the evening of day 1 (after surgery, approx. 8 - 9 h p.i.),

9) the next day,

10) on the following days up to cessation of the fluorescence (usually by day 3 in the normal skin and day 6 over the operated area).

PS maximum in the normal tissues was detected in $1-2 \mathrm{~h}$ p.i. Maximum tumor uptake-2 - $4 \mathrm{~h}$ p.i.

Optimal time for PDT of the experimental group of patients with the maximum tumor PS concentration and best contrast was found to be $4 \mathrm{~h}$ p.i.

\subsection{Efficacy Data Obtained in the Course of a 1-Month Observation}

The clinical studies have shown that in both groups of patients the therapy was performed radically, with no recurrence or regional metastases.

\subsubsection{Efficacy Data Obtained in the Course of Observation during the Period from Month 1 to Month 12}

The follow-up of the patients from both PDT + surgery and single surgery revealed the preservation of the $\mathrm{CR}$ in all of the patients.

\subsubsection{Efficacy Data Obtained in the Course of Observation during the Period from Year 1 to 5}

The CR was proven in all the patients of the PDT + surgery group, while only 9 out of 24 survived in the control group.

\subsubsection{Efficacy Data Obtained in the Course of Observation during the Period from Year 6 to 10}

The CR was proven in most of the patients of the PDT + surgery experimental group, with a few of 24 being lost from the study due to unknown reasons (not appeared), with only 3 out of 24 survivors found in the control group.

\section{Discussion}

Head and neck cancer is the sixth most common cancer worldwide, including basal (BCC) and squamous (SCC) cell carcinoma and melanoma.

In recent years, new therapeutic approaches have been developed for superficial BCC. It includes PDT [2] [4] and combinations of PDT with chemotherapy, e.g. using imiquimod, or radiation therapy [8].

Worldwide SCC occurrence was estimated 500,000 new cancer cases each year. Two out of every three patients are diagnosed with locally advanced disease, and for these, the recurrence rates have remained quite high at about $65 \%$ for the past forty years. PDT is a promising option for the treatment of this type of skin cancer as well. Effects of a combination of PDT with chemotherapy, for example, cisplatin, were quite promising [9].

As to melanoma, in case of the surgical treatment, the 5 -year survival is $40 \%$ by our study, that well correlates with data published in Russia [10].

In case metastases were found by the date of operation, the 5-year survival 
rate drops to $10 \%[11]$.

Using radiation therapy prior to surgery rises the rates, correspondingly, to $52 \%$ and $17 \%$.

Chemotherapy efficiency does not exceed 7.5\% - 30\% [12].

BC's effect is based on its ability to selectively accumulate in skin tumor after its intravenous administration and, during exposure to light at a wavelength corresponding to one of the absorption peaks of the drug (i.e., 406, 506, 536, 608 or $662 \mathrm{~nm}$ ), generate singlet oxygen that causes toxic effect on tumor cells and modifies their plasmatic membranes.

Comparing to protoporphyrin IX (metabolite of $\delta$-aminolevulinic acid and its esters), HPD, "Photofrin II", radahlorin has an intensive absorption band in the medium red part of the spectrum (662 - $664 \mathrm{~nm}$ ), where biological tissues are transparent to a considerable extent. It also gives an intensive fluorescence peak at $668 \mathrm{~nm}$ that is helpful for fluorescent diagnosis (PDD).

A comparatively higher therapeutic ratio and ROS production rate are observed for radahlorin as well, thus PDT with this PS is less painful, and the pain can easier be coped with analgetics. Radahlorin PDT is also more applicable for thick or large lesions (Figure 1, Figure 2), and as well for the areas where due to surface features the light spot is not homogenous or where there are many nerve endings (ears, around eyes, nose) (Figure 3).

In general, one can expect some benefits from Bremachlorin PDT vs. haemato- and protoporphyrin-IX-based agents (Levulan, ALA, Alasens, Metvix,

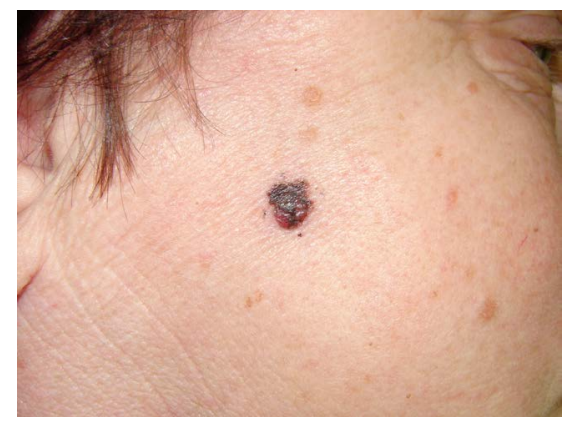

(a)

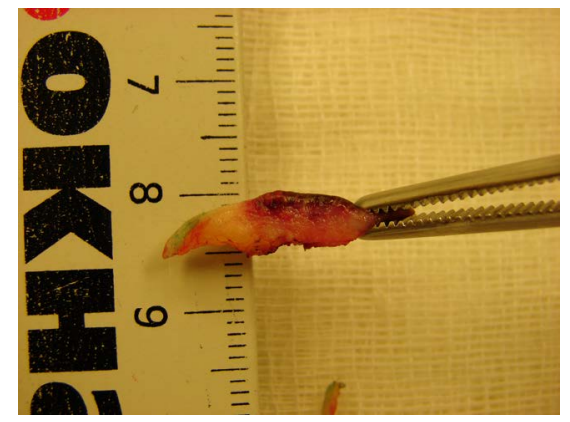

(b)

Figure 1. (a) Melanoma of the skin before PDT with photosensitizer Bremachlorin in a combination with surgery. (b) Melanoma after removal: the depth of 662-nm-laser light penetration and corresponding infiltrate are well seen to be around $6 \mathrm{~mm}$. 


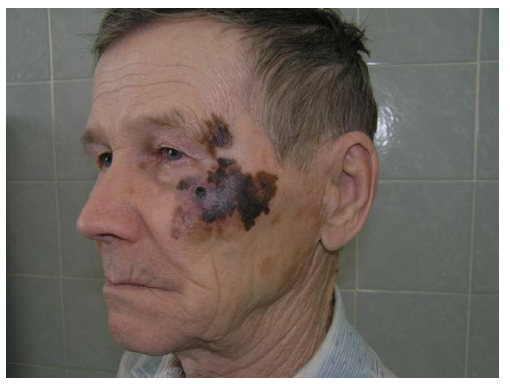

(a)

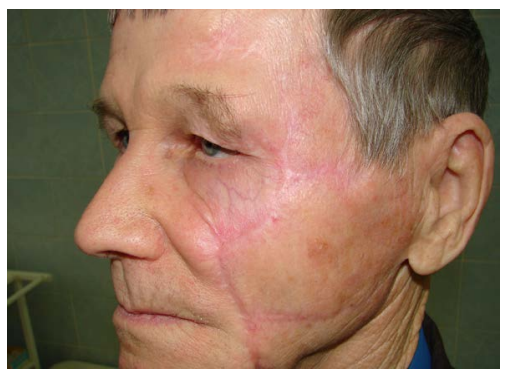

(b)

Figure 2. (a) A 80-year-old patient having melanoma of the skin with a 30-year backgroung of Dubreuilh's melanosis. (b) In 1 year after PDT combined with surgical resection and a plastic operation. Five year cancer free follow-up.

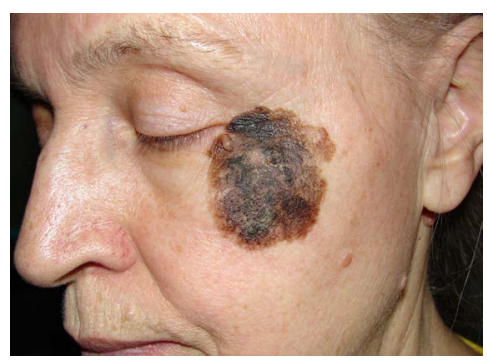

(a)

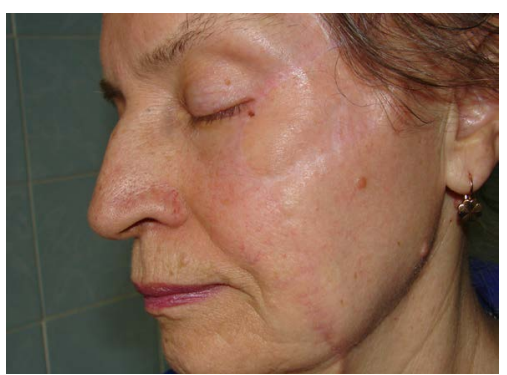

(b)

Figure 3. (a) Before PDT. (b) In 1 year after PDT combined with surgical resection and a plastic operation.

Hexvix, Photofrin II) PDT owing to better pharmaceutical, spectral, pharmacokinetic, toxicological, and energy performance of the former.

By the present study we have shown that combining surgery with PDT performed prior to resection the efficiency of the former may go up more than twice. The obligatory condition for such a rise in the effect seems to be using the 
$2^{\text {nd }}$ and $3^{\text {rd }}$ generation PSs with effective depth of necrosis exceeding $3 \mathrm{~mm}$, invasion level not more than 3 , and absence of local and distant metastases.

\section{Conclusion}

Conducting PDT one day prior to tumor dissection can guarantee to meet the ablastics principles at the subsequent surgery. It is reached on one hand due to the devitalization of cancer cells during PDT, and on the other hand, because of the thrombosis of the blood vessels supplying the tumor, which appear in the course of the photochemical reaction occurring in the tumor and surrounding tissues [13]. Besides, the PDT + surgery protocol can allow for dissecting the tumor with less distance from its edge, which seems to be important at the head and neck localizations of tumors. Further clinical studies towards combining PDT using the $2^{\text {nd }}$ generation PSs with chemotherapy and radiation therapy to treat melanoma of the skin are greatly challenging.

\section{Acknowledgements}

The authors would like to thank Immunolume Limited (1 Williams Road, Paihia 0200 New Zealand) for the support in publishing this article.

\section{References}

[1] Reshetnickov, A.V., et al. (2001) Photosensitizer, and Method Therefore. Patents of South Africa-2003/8407, Australia-2002212867, New Zealand-528237, United Kingdom-GB2389531, Norway-327751, Sweden-01981209.8, Italy-70446-BE/2009, Slovenia, The Netherlands, Rep. of Ireland, France, Germany, Switzerland, Belgium-1380295. Assignee: RADA-PHARMA International B.V., The Netherlands, Priority of 04.10.2001.

[2] Cohen, D.K. and Lee, P.K. (2016) Photodynamic Therapy for Non-Melanoma Skin Cancers. Cancers, 8, 90. https://doi.org/10.3390/cancers8100090

[3] Voet, M.A. (2004) Photodynamic Therapy for Pre-Melanomas. US Pat.6,723,750. Assignee: Allergan Inc., Irvine, CA, Priority of 20.04.2004.

[4] Kochneva, E.V., Filonenko, E.V., Vakulovskaya, E.G., Scherbakova, E.G., Seliverstov, O.V., Markichev, N.A. and Reshetnickov, A.V. (2010) Photosensitizer Radachlorin $^{\circledR}$ : Skin Cancer PDT Phase II Clinical Trials. Photodiagnosis and Photodynamic Therapy, 7, 258-267. https://doi.org/10.1016/j.pdpdt.2010.07.006

[5] Reshetnickov, A.V. and Mead, M.N. (2013) The Medicine of Light. The $1^{\text {st }}$ Edition, Amazon Press, USA, 255 p. (2014) The $2^{\text {nd }}$ Edition, Pumbo, The Netherlands, 271 p. (C) by Photoimmune Discoveries B.V. http://www.photoimmune.org/

[6] Douillard, S, Olivier, D, Patrice, T. (2009) In Vitro and In Vivo Evaluation of Radachlorin Sensitizer for Photodynamic Therapy. Photochemical \& Photobiological Sciences, 8, 405-413. https://doi.org/10.1039/b817175k

[7] Markichev, N.A., Eliseyenko, V.I., Alexeyev, Y.V., Armichev, A.V. and Reshetnickov, A.V. (2004) Photodynamic Therapy of Cutaneous Basal-Cell Carcinoma Using Photosensitizer from the Chlorins Family Radachlorin. Laser Medicine, 8, 144-145. (in Russian)

[8] Peris, K., Tambone, S., Kostaki, D., Varrassi, E. and Fargnoli, M.C. (2014) Treatments of Advanced Basal Cell Carcinoma: A Review of the Literature. Giornale Ita- 
liano Di Dermatologia E Venereologia, 151, 77-86.

[9] Ahn, J.C., Biswas, R., Mondal, A., Lee, Y.K. and Chung, P.S. (2013) Cisplatin Enhances the Efficacy of 5-Aminolevulinic Acid Mediated Photodynamic Therapy in Human Head and Neck Squamous Cell Carcinoma. General Physiology and Biophysics, 33, 53-62. https://doi.org/10.4149/gpb_2013046

[10] Paches, A.I. (2000) Head and Neck Tumors. Medicine, Moscow, Russia (in Russian).

[11] Demidov, L.V., Kharkevich, G.Yu. (2003) Skin Melanoma: Staging, Diagnostics and Treatment. The Russian Medical Journal, 11, 41-43. (in Russian).

[12] Bulat, Yu.V. (2001) The Contemporary Solutions to the Chemotherapy of the Disseminated Skin Melanoma. Moscow, Russia. (in Russian).

[13] Fyodorov, S.N., Kopaeva, V.G., et al. (1996) Vascular Effects of PDT. CIS Selected Papers: Laser Use in Oncology, SPIE, Proceedings Vol. 2728. 